\title{
SOBRE PRINCIPIOS Y REGLAS
}

\section{Introducción}

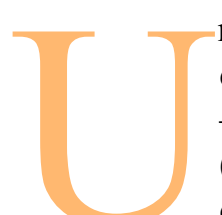
no de los temas más profusamente discutidos en la teoría del Derecho de las dos últimas décadas es, sin ninguna duda, el de los principios. El debate -como todo el mundo sabe- se inicia con un trabajo de Dworkin de 1967 (Dworkin, 1978), si bien los "principios jurídicos" (o los "principios generales del Derecho") son viejos conocidos (aunque quizá nada más que conocidos) de los juristas. De principios generales del Derecho hablaba ya el código civil austriaco de 1811, y siguieron hablando luego -entre muchos otros textos legislativos- el código civil italiano de 1865, el español de 1889 y el art. 38 del Estatuto del Tribunal Internacional de Justicia de la Haya; en los trabajos de dogmática jurídica y en las decisiones jurisprudenciales, la referencia a los principios es, puede decirse, un lugar común; y la reflexión sobre los mismos no ha estado tampoco ausente de la teoría o filosofía del Derecho anterior a Dworkin (cfr., por ejemplo, Del Vecchio, 1958; Esser, 1956; Bobbio, 1966). Si, a pesar de esto último, el planteamiento de Dworkin ha sido considerado en general como innovador, ello parece deberse (dejando a un lado los méritos intrínsecos de su obra) a su propósito de oponerse a la concepción dominante en la teoría del Derecho del momento y que él identifica correctamente con la obra de H. L. A. Hart.

Nosotros consideramos, por cierto, que en una buena medida el ataque de Dworkin yerra en el blanco, por la sencilla razón de que caracteriza mal la concepción de Hart y la del positivismo jurídico en general (cfr. Carrió, 1986). Nos parece igualmente que quien sostiene una concepción del Derecho como la de Hart no tiene por qué verse abocado a negar que el Derecho está integrado, además de por reglas, por pautas del tipo que Dworkin llama principios (o, al menos, no tendría mayor inconveniente en afirmar que muchas de esas pautas sí que forman parte, de 
acuerdo con los criterios hartianos, del Derecho). Pero no podemos dejar de reconocer también que los principios del Derecho - lo que quiera que sean- han sido cuando menos postergados en el análisis llevado a cabo por Hart, a pesar de ser de una enorme importancia para entender la estructura y el funcionamiento del Derecho.

Por otro lado, lo que vale para Hart se aplica también -y en mayor medida- a autores como Kelsen y Alchourrón y Bulygin, cuyas obras, en nuestra opinión, constituyen los hitos fundamentales de la teoría del Derecho inmediatamente anterior y posterior a $E l$ concepto de Derecho. En el caso de Alchourrón y Bulygin, parece que por "principios jurídicos" tan sólo podría entenderse una de estas dos cosas: O bien se trataría de normas no coactivas (esto es, enunciados que ordenan, prohíben o permiten conductas en determinados casos genéricos sin establecer sanciones), que se caracterizarían porque la descripción de las propiedades relevantes del caso genérico correspondiente presentaría un alto grado de generalidad y/o de indeterminación semántica; y en tal caso serían normas (enunciados que correlacionan casos con soluciones) indistinguibles de las reglas ${ }^{1}$. O bien podría tratarse de enunciados que ni serían normas ni tendrían influencia alguna sobre las consecuencias normativas del sistema (un ejemplo de ello, para estos autores, lo constituye los «enunciados que presentan teorías políticas» [Alchourrón y Bulygin, 1974, p. 107] y que, en realidad, no suscitan ningún problema de interés: «si tales enunciados son considerados parte integrante del sistema jurídico o no, no es una cuestión tan importante; Kelsen se inclina a eliminarlos del todo, lo cual parece ser bastante razonable» [ibid.]. Por lo que luego se verá, ninguna de estas dos alternativas -los principios, o son reglas o son irrelevantesparece aceptable.

Nuestro propósito en este trabajo no es el de presentar una versión mejorada de alguna de las anteriores concepciones del Derecho para así dar cuenta cabal de los principios. Tampoco pretendemos propiamente intervenir en una polémica que en muchos aspectos se ha vuelto ciertamente espesa. Lo que nos proponemos -eso sí, utilizando con libertad elementos surgidos en el anterior debate- es más bien tratar de dar una respuesta -o el comienzo de una respuesta- a algunas de las preguntas fundamentales que suscitan los principios en el Derecho: en primer lugar, qué rasgos estructurales diferencian a los principios de las reglas; en segundo lugar, qué tipo de razones para la acción son

\footnotetext{
${ }^{1}$ Utilizamos los términos «regla» $\mathrm{y}$ «principio» como especies del género «norma».
} 
los principios y en qué se diferencian a este respecto de las reglas; en tercer lugar, qué aportan los principios a la explicación y justificación en el ámbito jurídico.

\section{Tipos de principios}

\subsection{Diversos sentidos de "principio jurídico"}

Por empezar por lo obvio, es bien sabido que los juristas utilizan la expresión "principios jurídicos", "principios generales del Derecho" u otras semejantes con significados diversos, y no parece que tenga mucho sentido afirmar que uno de ellos es el esencial, el verdadero, o cosa por el estilo. De todas formas, las acepciones más significativas que se encuentran en contextos jurídicos parecen ser las siguientes (tomamos en cuenta, fundamentalmente, los análisis de Carrió, 1986 y Guastini, 1990):

a) "Principio" en el sentido de norma muy general, entendiendo por tal -como antes se apuntó- las que regulan un caso cuyas propiedades relevantes son muy generales. Por ejemplo, el art. 1091 del código civil español, cuando establece que «las obligaciones que nacen de los contratos tienen fuerza de ley entre las partes contratantes, y deben cumplirse al tenor de los mismos». Ni que decir tiene que la propiedad de que una norma sea general es cuestión relativa y graduable: la anterior norma -o principio- es más general que las que se aplican exclusivamente a los contratos de arrendamiento (por ejemplo, el art. 1545: «los bienes fungibles que se consumen con el uso no pueden ser materia de este contrato», y menos general que las que se aplican a los contratos y otros actos jurídicos (por ejemplo, el art. 11,1: «las formas y solemnidades de los contratos, testamentos y demás actos jurídicos se regirán por la Ley del país en que se otorguen»). La generalidad de una norma no es, en el sentido en que aquí empleamos la expresión, una cualidad que tenga que ver con la amplitud mayor o menor de la clase de los destinatarios de la misma, sino con la mayor o menor generalidad (o "genericidad", si se quiere) de las propiedades relevantes del caso que regula.

b) "Principio" en el sentido de norma redactada en términos particularmente vagos, como el art. 7, ap. 2 del código civil español: «la Ley no ampara el abuso del derecho o el ejercicio antisocial del mismo». Por supuesto, hay muchas normas que son vagas en el sentido de que en la descripción del caso genérico aparecen términos que tienen una periferia de textura abierta: el 
grueso de los casos ordinarios caen claramente dentro o fuera de la norma, pero existen también supuestos (excepcionales) en los que es dudoso si se les aplica o no la norma. Sin embargo, el ejemplo de norma (o de principio) indicado alude a otro tipo de vaguedad que se produce cuando se utilizan lo que los juristas llaman conceptos jurídicos indeterminados, esto es, términos (por ejemplo, "abuso del derecho") que son centralmente vagos y que no tienen sólo una periferia de textura abierta: también aquí pueden encontrarse casos claramente cubiertos o claramente fuera del alcance de la norma, pero la inmensa mayoría de los casos reales exige una tarea de concreción, esto es, una ponderación de factores relevantes cuya identidad y posibles combinaciones no resultan anticipables.

c) "Principio" en el sentido de norma programática o directriz, esto es, de norma que estipula la obligación de perseguir determinados fines. Por ejemplo, el art. 51 de la Constitución española: «los poderes públicos garantizarán la defensa de los consumidores y usuarios, protegiendo, mediante procedimientos eficaces, la seguridad, la salud y los legítimos intereses económicos de los mismos».

d) "Principio" en el sentido de norma que expresa los valores superiores de un ordenamiento jurídico (y que son el reflejo de una determinada forma de vida), de un sector del mismo, de una institución, etc. Por ejemplo, el art. 14 de la Constitución española: «los españoles son iguales ante la ley, sin que pueda prevalecer discriminación alguna por razón de nacimiento, raza, sexo, religión, opinión o cualquier otra condición o circunstancia personal o social».

e) "Principio" en el sentido de norma especialmente importante, aunque su grado de generalidad pueda ser relativamente bajo. Por ejemplo, el art. 56 ap. 3 de la Constitución española: «la persona del Rey es inviolable y no está sujeta a responsabilidad».

f) "Principio" en el sentido de norma de elevada jerarquía. Según este significado, todas las normas constitucionales serían principios, incluyendo las que no parecen ser ni muy generales ni muy importantes. Por ejemplo, el art. 160 de la Constitución española: «el Presidente del Tribunal Constitucional será nombrado entre sus miembros por el Rey, a propuesta del mismo Tribunal en pleno y por un período de tres años».

g) "Principio" en el sentido de norma dirigida a los órganos de aplicación jurídicos y que señala, con carácter general, cómo se debe seleccionar la norma aplicable, interpretarla, etc. Por ejemplo, el art. 4, ap. 2 del código civil: «las leyes penales, las excepcionales y las de ámbito temporal no se aplicarán a su- 
puestos ni en momentos distintos de los comprendidos expresamente en ellas».

h) "Principio" en el sentido de regula iuris, esto es, de enunciado o máxima de la ciencia jurídica de un considerable grado de generalidad y que permite la sistematización del ordenamiento jurídico o de un sector del mismo. Tales principios pueden o no estar incorporados al Derecho positivo. Ejemplo de lo primero es (al menos en el Derecho español) el principio de jerarquía normativa (en el art. 9,3 de la Constitución). De lo segundo, el principio del legislador racional que, aún sin estar formulado explícitamente en nuestro Derecho, es utilizado en numerosas ocasiones por los juristas teóricos o prácticos, por ejemplo, para defender que una norma debe interpretarse de una determinada manera (pues sólo así podría sostenerse que el legislador persiguió algún propósito racional al dictarla).

\subsection{Una propuesta de clasificación}

Esta lista de significados no tiene carácter exhaustivo (Carrió distingue -sin pretensiones de agotar los sentidos en que los juristas hablan de "principios"- once acepciones), y no es tampoco excluyente: los anteriores significados se solapan entre sí y hay ocasiones en que una misma norma (por ejemplo, el ya mencionado art. 51 de la Constitución española) podría servir como ejemplo de principio prácticamente en todas las acepciones indicadas. Para evitar, por lo menos en parte, las imprecisiones a que inevitablemente lleva el manejo de un término tan ambiguo, partiremos de esta triple clasificación de los principios.

2.2.1. Por un lado, se puede distinguir entre principios en sentido estricto ("principios" en la acepción indicada en d» y directrices o normas programáticas ("principios" en la aceptación indicada en c)). Esta distinción tiene, a nuestro juicio, carácter exhaustivo y excluyente. El que sea exhaustiva no implica, por cierto, prescindir de las otras acepciones de "principio" que se acaban de indicar, sino proceder a una redefinición, en el siguiente sentido: las características indicadas en a), b), e) y f) acompañan normalmente a los principios en sentido estricto y a las directrices, pero ninguna de ellas, ni el conjunto de todas ellas, por sí solas, permite calificar a una determinada pauta como principio; los principios en la acepción g) y h) son, si cumplen además las características a), b), e) y f), reconducibles a principios en sentido estricto o a directrices. Por otro lado, la distinción es 
excluyente pues, aunque es posible que un mismo enunciado pueda considerarse en ciertos contextos argumentativos como principio y en otros como directriz (y hasta podría decirse que ello constituye una ambigüedad característica de muchos principios), un mismo jurista no puede utilizarlo, en un mismo contexto argumentativo, como ambas cosas a la vez.

2.2.2. La segunda distinción que nos parece importante es la que puede trazarse (utilizando libremente terminología de Alchourrón y Bulygin con un alcance algo distinto del que tiene en estos autores) entre principios en el contexto del sistema primario o sistema del súbdito y principios en el contexto del sistema secundario o sistema del juez (y, en general, de los órganos jurídicos); esto es, entre los principios (pautas de comportamiento formulables como principios en sentido estricto o como normas programáticas) en cuanto dirigidos a guiar la conducta de la gente que no consiste en el ejercicio de poderes normativos (conducta no normativa, diremos en adelante para simplificar) y los principios en cuanto dirigidos a guiar el ejercicio de poderes normativos (la creación o aplicación de normas) de los órganos de producción jurídica. Esta distinción es exhaustiva, pero no excluyente. Hay, sin duda, principios cuya virtualidad se limita a guiar el ejercicio de poderes normativos, pero no principios que guíen sólo conductas no normativas: todos los principios de los que puede predicarse que guían la conducta no normativa de los destinatarios de las normas jurídicas en general, guían también la conducta normativa de, al menos, los órganos de aplicación.

2.2.3. Finalmente, la última distinción que nos interesa es la que media entre principios explícitos, esto es, principios formulados expresamente en el ordenamiento jurídico, y principios implícitos, esto es, principios extraídos a partir de enunciados presentes en el ordenamiento jurídico (por ejemplo, el principio de que las normas han de interpretarse como si las hubiera dictado un legislador racional). Esta distinción, obviamente, es exhaustiva y excluyente.

\section{Principios y reglas}

Además de estas distinciones de carácter interno, los principios deben distinguirse hacia afuera (externamente) de otras pautas de comportamiento que integran un Derecho. Aquí partiremos de la idea de que los Derechos están formados por normas (y por otras 
entidades de las que aquí no nos ocuparemos, como las definiciones) y que las normas pueden, a su vez, ser reglas o principios.

El problema, por tanto, puede plantearse en estos términos: ¿cómo se pueden distinguir los principios de las reglas y, simultáneamente, cuál es el alcance que tienen las anteriores clasificaciones de los principios? Nuestra estrategia para contestar a esta pregunta consistirá en partir de dos enfoques característicos que suelen adoptarse en relación con las normas y que nosotros proyectaremos al problema que nos ocupa. Al primero de estos enfoques podría llamársele estructural, pues consiste en ver las normas como entidades organizadas de una cierta forma. Un ejemplo de ello es la concepción de las normas que encontramos en Normative Systems, esto es, como correlaciones entre casos genéricos (conjuntos de propiedades) y soluciones (esto es, la calificación normativa de una determinada conducta). La otra forma característica de entender las normas podría llamarse funcional, pues se centra en el papel o la función que las mismas cumplen en el razonamiento práctico de sus destinatarios. Un ejemplo de este enfoque, en la teoría del Derecho contemporánea, lo ofrece de forma muy destacada la obra de Raz. En dicha obra, como se sabe, las normas son vistas como razones para la acción; lo que interesa básicamente, desde esta perspectiva, es mostrar qué tipo de razones son las normas, y cómo operan en el razonamiento práctico. La cuestión de cómo distinguir los principios de las reglas o, entre sí, los diversos tipos de principios antes señalados, podría adquirir tonos diferentes, según que se consideren las normas desde una u otra perspectiva.

\subsection{Un enfoque estructural de la distinción}

Desde el primero de los enfoques, el problema parece ser éste: aceptado que las reglas pueden formularse siempre recurriendo a un esquema condicional, esto es, de correlación caso/solución (lo que no significa aceptar -ni negar- que las normas sean entidades lingüísticas, sino tan sólo que pueden expresarse en un lenguaje) ¿puede decirse lo mismo de los principios? En realidad, antes de contestar a esta pregunta es preciso plantearse otra de carácter previo, esto es, la de cuáles de las anteriores clasificaciones de los principios son relevantes a los efectos que ahora nos interesan. Parece claro que no lo es ni la distinción entre principios explícitos e implícitos (por lo que se acaba de decir), ni entre principios en el contexto del sistema primario y principios 
en el contexto del sistema secundario (si existiera alguna diferencia estructural por el hecho de que el caso regulado por una norma consista o haga referencia a su vez a normas, es lógico pensar que esto se aplicaría tanto a los principios como a las reglas). Así pues, lo que se trata de ver es si los principios en sentido estricto y las normas programáticas tienen o no una estructura condicional (esto es, correlacionan un caso con una solución normativa) y/o si los operadores deónticos que rigen para las reglas son los mismos que los de los principios.

3.1.1. En nuestra opinión, los principios en sentido estricto pueden formularse siempre como enunciados que correlacionan casos con soluciones, pero eso no quiere decir que, desde esta perspectiva, no exista ninguna diferencia entre reglas y principios. La diferencia estriba en que los principios configuran el caso de forma abierta, mientras que las reglas lo hacen de forma cerrada. Con ello queremos decir que mientras que en las reglas las propiedades que conforman el caso constituyen un conjunto cerrado, en los principios no puede formularse una lista cerrada de las mismas: no se trata sólo de que las propiedades que constituyen las condiciones de aplicación tengan una periferia mayor o menor de vaguedad, sino de que tales condiciones no se encuentran siquiera genéricamente determinadas. El tipo de indeterminación que aqueja a los principios es, pues, más radical que el de las reglas (aunque, desde luego, entre uno y otro tipo de indeterminación puede haber casos de penumbra).

Robert Alexy, desarrollando, por lo demás, algo que ya estaba presente en Dworkin, ha escrito que «el punto decisivo para la distinción entre reglas y principios es que los principios son normas que ordenan que se realice algo en la mayor medida posible, en relación con las posibilidades jurídicas y fácticas. Los principios son, por consiguiente, mandatos de optimización que se caracterizan porque pueden ser cumplidos en diversos grados y porque la medida ordenada de su cumplimiento no sólo depende de las posibilidades fácticas, sino también de las posibilidades jurídicas. El campo de las posibilidades jurídicas está determinado a través de principios y reglas que juegan en sentido contrario. En cambio, las reglas son normas que exigen un cumplimiento pleno y, en esta medida, pueden siempre ser sólo cumplidas o incumplidas. Si una regla es válida, entonces es obligatorio hacer precisamente lo que ordena, ni más ni menos» (Alexy, 1988, pp. 143-4). A nuestro juicio, que los principios puedan ser cumplidos en diversos grados es verdadero por lo que se refiere a las directrices o normas programáticas, pero no lo es en el caso de los principios 
en sentido estricto. Veámoslo mediante algunos ejemplos.

Un principio como el formulado en el art. 14 de la Constitución española, visto como principio secundario, puede, nos parece, presentarse en forma de un enunciado condicional como el siguiente: «si un órgano jurídico ha de dictar una norma, aplicarla, etc., y no se dan circunstancias normativas o fácticas que exijan otra cosa (esto es, que desplacen al principio), a dicho órgano le está prohibido establecer o hacer que prevalezca discriminación alguna por razón de raza, sexo, religión, opinión o cualquier otra condición o circunstancia personal o social». La indeterminación característica de los principios la encontramos aquí únicamente en la configuración abierta de las condiciones de aplicación, pero no en la descripción de la conducta prohibida: discriminar. Puede, desde luego, entenderse que "discriminación" es un término vago en ciertos contextos, pero este tipo de vaguedad se da también en las pautas a las que llamamos "reglas". La regla de que la mujer trabajadora debe recibir igual salario que el hombre se diferencia del principio anterior únicamente en que sus condiciones de aplicación se configuran de forma cerrada (así, el art. 28 del Estatuto de los Trabajadores establece que «el empresario está obligado a pagar por la prestación de un trabajo igual el mismo salario, tanto por salario base como por los complementos salariales, sin discriminación alguna por razón de sexo»), pero también aquí puede haber problemas de vaguedad a la hora de establecer tanto si sus condiciones de aplicación se dan en un determinado caso individual (pueden existir dudas sobre si una determinada actividad debe considerarse o no como "trabajo"), o sobre el alcance de la descripción de la conducta prohibida (un complemento salarial para vestuario diferente por razón del sexo ¿debe considerarse prohibido por dicho artículo 28?). Estructuralmente, la única diferencia entre el art. $14 \mathrm{CE}$ y el art. 28 ET reside en que, en el último caso, las condiciones de aplicación de la norma constituyen un conjunto cerrado, aunque su formulación pueda presentar problemas de indeterminación semántica (e insistimos en que las indeterminaciones semánticas no afectan al carácter de regla, a no ser que alcance un grado extremo que no permita que se hable ya de determinación de un modelo de conducta). Pero en cuanto a la descripción del modelo de conducta calificado deónticamente, ambas normas presentan un grado de determinación semejante. Dicho de otra forma: si se ha determinado que el principio de igualdad consagrado en el art. 14 prevalece, en la combinación de factores relevantes que presenta un determinado caso individual, frente a principios o reglas que jueguen en sentido contrario, este principio exige un cumplimiento 
pleno: o se cumple o no se cumple, pero no caben modalidades graduables de cumplimiento.

3.1.2. Si de los principios en sentido estricto pasamos a las directrices o normas programáticas, las cosas parecen ser algo distintas. Una norma como, por ejemplo, el art. 51 de la Constitución antes recordado puede también expresarse siempre en forma de un enunciado condicional: «si x es un poder público y no se dan circunstancias normativas o fácticas que lo impidan, entonces $\mathrm{x}$ debe utilizar los procedimientos eficaces tendentes a proteger la seguridad, la salud y los legítimos intereses económicos de los consumidores y usuarios». Pero si ahora nos planteamos la cuestión de en qué se diferencian estructuralmente el art. 51 y el art. $14 \mathrm{CE}$, resulta obvio que mientras el último de ellos sólo configura de forma abierta sus condiciones de aplicación, pero no el modelo de conducta calificado deónticamente, el primero configura de forma abierta tanto las condiciones de aplicación como el modelo de conducta prescrito. La diferencia, pues, entre un principio en sentido estricto y una directriz, desde la perspectiva desde la que estamos ahora contemplando las normas, parece ser la siguiente: de los principios en sentido estricto cabe decir que son mandatos de optimización únicamente en el sentido de que, al estar configuradas de forma abierta sus condiciones de aplicación, la determinación de su prevalencia o no en un caso individual determinado exige su ponderación, en relación con los factores relevantes que el caso presente, con principios y reglas que jueguen en sentido contrario; pero una vez determinado que en ese caso prevalece el principio, éste exige un cumplimiento pleno. Las directrices, por el contrario, al estipular la obligatoriedad de utilizar medios idóneos para perseguir un determinado fin, dejan también abierto el modelo de conducta prescrito: las directrices sí pueden, en efecto, ser cumplidas en diversos grados. Esto es lo que explica que, por ejemplo, el art. 14 de la Constitución cambiaría de sentido si se estableciera como una norma programática («los poderes públicos promoverán la igualdad de los españoles ante la ley, para que no pueda prevalecer discriminación alguna por razón de nacimiento, raza...»).

\subsection{Principios y reglas como razones para la acción}

La otra forma de entender las normas -como razones para la acción- es probablemente más iluminadora que la anterior, referida al tema de los principios. Para facilitar el análisis, empezaremos 
por considerar únicamente los principios en cuanto pautas dirigidas a las autoridades normativas y, más en concreto, a los órganos jurisdiccionales entendidos en sentido amplio (lo que Raz llama "órganos primarios"): aquellos órganos a quienes el propio Derecho confiere el poder normativo para resolver autoritativamente las disputas y les impone el deber de hacerlo jurídicamente (fundamentando sus resoluciones en las pautas identificadas como jurídicas). Nos olvidamos también por el momento de la distinción entre principios en sentido estricto y directrices.

3.2.1. Pues bien, en este contexto, la distinción -y las relaciones- entre reglas, principios explícitos y principios implícitos aparecen estrechamente asociada a la distinción -y a las relaciones- entre obediencia a las autoridades normativas y propia deliberación en la conducta jurídicamente guiada de los órganos jurisdiccionales.

3.2.1.1. Tomando como punto de partida una conocida caracterización del último Hart (1982), cabe considerar a las reglas jurídicas de mandato ${ }^{2}$ como razones para la acción perentorias e independientes del contenido. "Razones perentorias" quiere decir lo mismo que "razones protegidas" (en la terminología de Raz), a saber, que constituyen una razón de primer orden para realizar la acción exigida -en el contexto que nos interesa, para dictar una resolución cuyo contenido corresponda al de la regla- y una razón de segundo orden para «excluir o suprimir cualquier deliberación independiente por parte de su destinatario sobre los argumentos en pro y en contra de realizar la acción» -en el mismo contexto, para excluir que el contenido de la resolución se fundamente en la apreciación por parte del órgano jurisdiccional de cuál fuera, atendiendo a los méritos del caso, la mejor resolución a dictar. Las reglas están destinadas, pues, a que, cuando se dan sus condiciones de aplicación, los órganos jurisdiccionales excluyan, en cuanto base de su resolución, su propio juicio acerca del balance de razones aplicables y adopten como tal base el contenido de la regla. El carácter independiente del contenido afecta al por qué los órganos jurisdiccionales deben obedecer las reglas, esto es, a las razones por las que deben considerarlas como razones "perentorias" o "protegidas". Y en este sentido, se supone que los órganos jurisdiccionales deben

${ }^{2}$ Las reglas permisivas quedan fuera de la cuestión porque no pueden constituir razones operativas. 
considerarlas así por razón de su fuente (esto es, de la autoridad normativa que las ha dictado o adoptado): su origen en dicha fuente es condición suficiente para que los órganos jurisdiccionales deban considerarlas como razones perentorias.

3.2.1.2. De los principios explícitos cabe decir que son razones para la acción independientes del contenido, pero no perentorias. Son independientes del contenido porque la razón por la que son razones para la acción de los órganos jurisdiccionales, esto es, la razón por la que deben entrar a formar parte del razonamiento justificatorio de las decisiones de los órganos jurisdiccionales es la misma que en el caso de las reglas: a saber, su origen en una determinada fuente. No son, sin embargo, razones perentorias porque no están destinadas a excluir la deliberación por parte del órgano jurisdiccional acerca del contenido de la resolución a dictar, sino que constituyen meramente razones de primer orden para resolver en un determinado sentido, cuya fuerza respecto de otras razones (otros principios) -que puedan constituir, a su vez, razones para resolver en otro sentido- ha de ser ponderada por el propio órgano jurisdiccional.

3.2.1.3. Los principios implícitos, a su vez, son razones para la acción que no son perentorias ni independientes del contenido. No son perentorias por la misma razón por la que no lo son los principios explícitos, y no son independientes del contenido porque si deben entrar a formar parte del razonamiento de los órganos jurisdiccionales no es por virtud de su origen en fuente alguna, sino por cierta cualidad de su contenido. En nuestra opinión, dicha cualidad no es su justicia intrínseca (esto es, los principios no se extraen sin más de la moral social, como a veces parece dar a entender Dworkin [1978 y 1986], ni son simplemente pautas que gozan de arraigo social, como sostienen algunos civilistas ilustres como Diez Picazo), sino su adecuación o coherencia en relación con las reglas y principios basados en fuentes.

Puede decirse, pues, que las reglas son razones autoritativas tanto en cuanto al por qué operan como al cómo operan en el razonamiento justificatorio de los órganos jurisdiccionales; que los principios explícitos son razones autoritativas en cuanto al por qué de su presencia en dicho razonamiento pero no en cuanto al cómo operan en él; y que los principios implícitos, en fin, no son autoritativos en cuanto a su forma de operar en el razonamiento 
y, en cuanto al por qué de su presencia en él, lo son, en todo caso, sólo indirectamente, en tanto que tal presencia se fundamenta en su adecuación a las reglas y principios explícitos.

3.2.2. Si de los principios secundarios pasamos ahora a los principios primarios, esto es, a los principios en cuanto pautas dirigidas a la gente en general, el análisis no tendría por qué variar mucho con respecto al anterior. La diferencia parece estribar más bien en que, a diferencia de los órganos jurisdiccionales, la gente -salvo en casos excepcionales- no tiene por qué justificar su comportamiento en relación con las normas jurídicas. Pero, por lo demás, las reglas -para quien las sigue- operan como razones perentorias o protegidas, mientras que los principios lo hacen como razones de primer orden que deben ser ponderadas con otras razones.

3.2.3. Por lo que se refiere a la contraposición entre principios en sentido estricto y directrices, consideramos que tal distinción, en términos de razones para la acción, podría trazarse como sigue. Las directrices generan razones para la acción de tipo instrumental o estratégico: el que la consecución de un fin F sea deseable hace que exista, en principio, una razón en favor de todo aquello que conduzca al fin; la razón no es excluyente, pues puede haber razones en sentido contrario y que tengan una mayor fuerza. Por el contrario, las razones para la acción que derivan de principios en sentido estricto son razones de corrección: al igual que las anteriores, no son tampoco excluyentes, pero, en la deliberación del sujeto, las razones de corrección operan como razones últimas (no son razones finalistas, sino razones finales). Por eso, las razones estratégicas que derivan de directrices pueden y deben ser evaluadas $-\mathrm{y}$, en su caso, superadas- por razones de principio, mientras que lo contrario no puede ocurrir: si se tiene una razón de principio para hacer $\mathrm{X}$, entonces el no hacer $\mathrm{X}$ sólo puede justificarse apelando a otras razones de corrección -basadas en principios- que tengan un mayor peso, pero no a razones finalistas -basadas en directricesque muestren que la consecución de un cierto fin es incompatible con la acción X.

\section{Dimensiones explicativa y justificatoria de los principios}

Pasemos ahora a considerar el tercero de los problemas indicados al comienzo. El punto de partida en este caso lo va a constituir la distinción entre las dos funciones básicas que, en 
nuestra opinión, desempeñan los principios en el Derecho: una función de explicación y otra de justificación.

\subsection{Los principios en la explicación del Derecho}

Plantearse el problema de hasta qué punto los principios permiten explicar lo que es el Derecho en general, un ordenamiento jurídico en particular o un sector del mismo, podría pensarse que significa tanto como situar el problema en el contexto de la ciencia del Derecho (con sus diversos niveles de abstracción). Los principios se verían, pues, como piezas o instrumentos que permiten dar cuenta de una determinada realidad (el Derecho visto desde diferentes ángulos o niveles de abstracción).

Pues bien, los principios cumplen con esta función explicativa al menos en estos dos sentidos. En primer lugar, por su capacidad para sintetizar una gran cantidad de información: la referencia a unos pocos principios nos permite entender cómo funciona una institución jurídica en el conjunto del ordenamiento jurídico, y en relación con el sistema social. Los principios son -como las leyes científicas- enunciados que hacen posible una descripción económica de una determinada realidad (en este caso, el Derecho), y cumplen por tanto una función didáctica -en sentido amplio- de una gran importancia. Pero, en segundo lugar $-\mathrm{y}$ esto es aún más importante- los principios nos permiten también entender el Derecho -o los diferentes Derechos- no como un simple conjunto de pautas, sino como un conjunto ordenado, esto es, como un conjunto dotado de sentido. Por ello, conocer los principios de una institución o de un determinado Derecho permite incluso, hasta un cierto punto, predecir cuáles son las soluciones a los problemas jurídicos fijadas en disposiciones específicas. Esta doble capacidad de los principios para presentar de una manera breve y ordenada un sector de (o todo) un ordenamiento jurídico no es ni más ni menos que lo que suele llamarse sistematización del Derecho. Y como ésta es la función principal que, se supone, ha de realizar la ciencia jurídica, queda claro que los principios juegan aquí un papel fundamental.

Sin pretender, por supuesto, negar esto, nos parece importante poner el énfasis en que la ciencia jurídica -o, más exactamente, lo que suele llamarse "dogmática jurídica"- es una disciplina normativa, pero no únicamente en el sentido obvio en que su objeto son normas, sino también en otros dos sentidos (probablemente igual de obvios, aunque no siempre se haya entendido así), a saber: en cuanto que utiliza un método normativo (el punto de 
vista de la dogmática no es el punto de vista externo en relación con las normas) y en cuanto cumple una función que podría llamarse "normativa", puesto que el dogmático no se limita a describir las normas vigentes, sino que propone o sugiere criterios para la resolución de problemas jurídicos. Para la dogmática, en nuestra opinión, la sistematización de un cierto material normativo es una tarea central, pero que no constituye un fin en sí misma, sino un medio para realizar lo que constituye su función social más relevante: suministrar criterios para la aplicación, interpretación y modificación del Derecho. En definitiva, lo que caracteriza a la dogmática no es tanto $-\mathrm{o}$, al menos, no es sólo- su función explicativa, sino su función de justificación.

\subsection{Los principios en el razonamiento jurídico}

Pasemos, pues, a ocuparnos de la dimensión justificatoria de los principios jurídicos o, dicho de otra manera, del papel que los mismos juegan en el razonamiento jurídico.

4.2.1. El razonamiento jurídico, en cuanto tipo especial de razonamiento práctico, es, por supuesto, una actividad compleja y que puede envolver elementos de muy diverso género. Ciñéndonos a su aspecto normativo, puede decirse que el papel de los principios contrasta con el de las reglas en cuanto que, desde una cierta perspectiva, su contribución a la argumentación es más modesta, mientras que, vistas las cosas desde otro punto de vista, cabría decir que los principios superan a las reglas.

Los principios son menos que las reglas en estos dos sentidos. Por un lado, no presentan las ventajas de las reglas, pues no permiten ahorrar tiempo a la hora de decidir un curso de acción. Si una regla es aceptada, entonces se evita tener que entrar en un proceso de ponderación de razones en pro y en contra; la regla opera, por tanto, como un elemento que reduce la complejidad de los procesos de argumentación. Sin embargo, los principios -como se ha visto- no eximen de la tarea de efectuar esa ponderación. Por otro lado, los principios, en cuanto premisas a utilizar en los argumentos prácticos, tienen menos fuerza (son menos concluyentes) que las reglas. Si alguien acepta como premisa de su razonamiento la regla "si se da X, entonces se debe hacer Y" y acepta también que "X ha tenido lugar", de ahí tiene que inferir necesariamente "se debe hacer Y". Sin embargo, de la premisa "F es un fin a alcanzar" y "G (aun cuando se trate de un comportamiento no prohibido por el ordenamiento jurídico) conduce a F" 
no puede pasarse a la conclusión "se debe hacer G", sino simplemente a la de "existe una razón para efectuar G"; lo mismo cabe decir en relación con las premisas "C es un tipo de comportamiento valioso" y "c es una acción del tipo C" que no permiten ir más allá de la conclusión "hay una razón para efectuar c".

Pero los principios son también más que las reglas, y en otros dos sentidos. Por un lado, porque al estar enunciados -o poder enunciarse- en términos más generales, entran también en juego en un mayor número de situaciones; esto es, al tener un mayor poder explicativo que las reglas, tienen también un mayor alcance justificatorio. Por otro lado, la menor fuerza de los principios en cuanto premisas del razonamiento práctico va aparejada a una mayor fuerza expansiva. Así, por ejemplo, a partir de las premisas "todos los varones físicamente aptos deberán hacer el servicio militar" y "si te intervienen quirúrgicamente pasarás a ser considerado físicamente apto" no se concluye "debes intervenirte quirúrgicamente" y ni siquiera "existe una razón para que te intervengan quirúrgicamente" (pues es posible que uno no desee hacer el servicio militar, de manera que, para él, ello no cuenta como razón). En cambio, a partir del principio de que "los españoles tienen derecho a una vivienda digna" junto con el enunciado "abaratar los créditos para viviendas facilita que un mayor número de personas acceda a una vivienda digna" sí que se concluye, cuando menos, que "hay una razón para que el Estado abarate los créditos para la compra de viviendas".

4.2.2. Las diferencias que se acaban de establecer entre la diversa forma de operar en el razonamiento jurídico reglas y principios parecen valer para los principios en general, esto es, no toman en consideración todavía la triple clasificación de los principios con que habíamos operado hasta ahora. Conviene, pues, analizar si esas distinciones son también relevantes -y en qué sentido lo son- desde el punto de vista del razonamiento jurídico. Veámoslo.

4.2.2.1 En primer lugar, parece que tiene que haber una diferencia entre principios secundarios y primarios, en la medida en que la distinción se entienda como referida a la utilización de los principios por parte de la gente en general o por parte de los órganos jurisdiccionales (el supuesto de utilización de los principios por parte de abogados, dogmáticos, etc., podría asimilarse -a estos efectos- a la argumentación jurisdiccional). Y la diferencia -como ya antes se apuntó- es que para los primeros los principios no constituyen generalmente más que 
guías para la acción, mientras que para los órganos jurisdiccionales (y para los abogados y dogmáticos) los principios -como el resto de las pautas del ordenamiento jurídico- deben servirles no solamente para resolver el problema de qué hacer, sino también el de cómo justificar lo que se ha hecho o se va a hacer, esto es, la toma de decisiones jurídicas.

A veces se supone que los principios sólo entran en juego en el razonamiento justificatorio de los órganos jurisdiccionales cuando éstos tienen que hacer frente a casos difíciles, pues en los casos fáciles las reglas son elementos necesarios y suficientes para justificar la decisión. Esta forma de ver las cosas no nos parece, sin embargo, aceptable, por lo siguiente. Un caso es fácil precisamente cuando la subsunción de unos determinados hechos bajo una determinada regla no resulta controvertible a la luz del sistema de principios que dotan de sentido a la institución o sector normativo de que se trate. $\mathrm{Y}$ "que dotan de sentido" puede tener, a su vez -como ya se ha indicado-, un doble sentido: puede tratarse de los valores cuya realización viene asegurada por el cumplimiento de la regla, o bien de los objetivos sociales para cuya consecución el cumplimiento de la regla aparece como medio. Pero, en uno u otro de los sentidos de "dotar de sentido", es a la luz de los principios explícitos o implícitos del sector normativo de que se trata como cabe determinar si un caso es fácil o difícil.

Lo anterior tiene importantes consecuencias, porque invalida una imagen muy extendida acerca de la manera como el Derecho guía la conducta de los órganos jurisdiccionales. Dicha imagen podría resumirse así: el Derecho guía primaria y centralmente tal conducta mediante reglas, esto es, mediante razones perentorias (razones -repetimospara dictar una resolución cuyo contenido esté basado en el de la regla y para excluir que opere ninguna otra razón como base del contenido de la resolución) y sólo requiere de la deliberación de los órganos jurisdiccionales en zonas periféricas: zonas periféricas originadas, bien por los déficits del lenguaje ordinario (casos que caen dentro del área de penumbra del significado de los términos empleados en la formulación de la regla), bien en el relativo desconocimiento de los hechos futuros y la relativa indeterminación de propósitos del legislador (supuestos de lagunas y antinomias normativas y también de atribución a los órganos jurisdiccionales de potestades discrecionales en la resolución de determinados casos). En esta concepción, lo central de la conducta jurídicamente guiada de los órganos jurisdiccionales es la obediencia a razones perentorias; la deliberación acerca de razones no perentorias es periférica o marginal.

Ahora bien, si la consideración de un caso como fácil, esto es, 
como subsumible en una determinada regla a adoptar como razón perentoria para la resolución del mismo, sólo puede hacerse teniendo en cuenta principios, entonces la dimensión de obediencia a razones perentorias ya no puede aparecer como primaria: la obediencia a tales razones exige la previa deliberación y sólo tiene lugar en el territorio acotado por ésta. Naturalmente, el Derecho no reconoce a cualquier razón válida como un integrante legítimo de tal deliberación: salvo que el Derecho faculte en algún caso al órgano jurisdiccional para atender a razones distintas de las que él mismo contiene, ha de tratarse de razones contenidas en el propio Derecho, esto es, de principios explícitos o implícitos. Puede hablarse así de una deliberación normativamente guiada que, si se quiere, constituye también una forma de "obediencia", pero -y esto es lo importante- sustancialmente diferente de la obediencia que consiste en el seguimiento de una razón perentoria.

Quizá todo lo anterior pueda resumirse diciendo que el Derecho constituye, para los órganos jurisdiccionales, un sistema excluyente en un doble nivel y en un doble sentido. En un primer sentido $-\mathrm{y}$ en un primer nivel- por cuanto que impone a tales órganos jurisdiccionales el deber de componer un balance de razones integrado únicamente por las constituidas por las pautas jurídicas, siendo admisible la toma en consideración de otras razones únicamente en la medida en que las propias pautas jurídicas lo permitan. En un segundo sentido $-\mathrm{y}$ en un segundo nivel- por cuanto que tal balance de razones remite, no en todos los casos pero sí en la mayoría, a adoptar como base de la resolución una regla jurídica, esto es, una razón perentoria. Cabría así dividir a los casos en dos grupos: aquellos cuya resolución se fundamenta en el balance de razones jurídicas que se integran en la deliberación del órgano jurisdiccional, y aquellos otros en los que tal balance de razones exige el abandono de la deliberación y la adopción como base de la resolución de una razón perentoria.

4.2.2.2. Por lo que se refiere a la distinción entre el uso argumentativo de principios en sentido estricto, y de directrices, lo fundamental ya ha quedado dicho con anterioridad, cuando contrapusimos las razones de corrección a las razones de carácter instrumental o estratégico. Ahora podemos añadir nuestro acuerdo con la afirmación de Summers (1978) de que las rightness reasons y las goal reasons constituyen los principales tipos de razones substantivas (que él contrapone a las razones autoritativas), las cuales, a su vez, vienen a ser el núcleo de la argumentación jurídica en los casos difíciles. 
4.2.2.3. Finalmente, en lo que concierne a la distinción entre principios explícitos e implícitos, no cabe duda de que los segundos suponen una mayor complejidad que los primeros, pues en relación con ellos no se plantea únicamente el problema de cómo deben ser usados en cuanto premisas de una argumentación jurídica, sino también el de cómo se justifica adoptarlos como tales premisas. En este sentido, nos parece que afirmar que "X es un principio implícito" de una determinada institución, sector normativo, o sistema jurídico es lo mismo que afirmar que las reglas y principios explícitos correspondientes son coherentes con $\mathrm{X}$ y que a tales reglas y principios explícitos, entendidos como formulaciones lingüísticas, debe adscribírseles un contenido proposicional que resulte coherente con $\mathrm{X}$. Pero el requisito de "coherencia" con el material normativo, como es sabido, puede en muchas ocasiones ser satisfecho tanto por el principio X como por otros principios. En este sentido, el problema de la identificación de principios jurídicos implícitos viene a coincidir con el problema general de la interpretación jurídica. Y ello queda ya fuera de los límites de este trabajo.

\section{BIBLIOGRAFÍA}

Alchourrón, Carlos E. y Bulygin, Eugenio: Introducción a la metodología de las ciencias jurídicas y sociales. Astrea, Buenos Aires, 1974. Edición inglesa, Normative Systems, Springer Verlag, Wien-New York, 1971.

Alexy, Robert: «Sistema jurídico, principios jurídicos y razón práctica», en Doxa. Cuadernos de Filosofía del Derecho, núm. 5, 1988.

Bobbio, Norberto: «Principi generali di diritto», en Novissimo Digesto Italiano, XIII, Torino, 1966.

Carrió, Genaro R.: «Principios jurídicos y positivismo jurídico», en Notas sobre Derecho y lenguaje, Abeledo Perrot, Buenos Aires, 1986 (3. a edición).

Del Vecchio, Giorgio: «Sui principi generali del diritto», en Studi sul diritto, vol. I, Milano, 1958.

Díez-Picazo, Luis y Gullón, Antonio: Sistema de Derecho civil, vol. I, 7. a ed. Tecnos, Madrid, 1989.

Dworkin, Ronald: Taking Rights Seriously. Duckworth, London, 1978. Dworkin, Ronald: Law's Empire. Fontana Press, London, 1986.

Esser, Joseph: Grundsatz und Norm in der richterlichen Forbildung des Privatrechts. J. C. B. Mohr, Ubingen, 1956.

Guastini, Riccardo: «Principi del diritto», en Dalle fonti alle norme, Giappichelli, torino, 1990.

Hart, Herbert L. A.: The Concept of Law, Oxford University Press, 1961. 
Hart, Herbert L. A.: «Commands and Authoritative Legal Reasons», en Essays on Bentham. Jurisprudence and Political Theory, Clarendon Press, Oxford, 1982.

Raz, Joseph: La autoridad del Derecho. Ensayos sobre Derecho y moral, trad. de Rolando Tamayo, UNAM, México, 1982.

Raz, Joseph: Practical Reason and Norms. Princeton University Press, 1990 (2. ${ }^{\mathrm{a}}$ ed.).

Summers, Robert S.: «Two Types of Substantive Reasons», en Cornell Law Review, n. ${ }^{\circ}$ 63, 1978. 